\title{
A Defect Localization Approach Based on Improved Areal Coordinates and Machine Learning
}

\author{
Dandan Pang $\mathbb{D}^{1,2}$ Yongqing Jiang, ${ }^{1,2}$ Yukang Cao, ${ }^{1,2}$ and Baozhu Li $\mathbb{D}^{3}$ \\ ${ }^{1}$ School of Information and Electrical Engineering, Shandong Jianzhu University, Jinan 250101, China \\ ${ }^{2}$ Shandong Key Laboratory of Intelligent Buildings Technology, Shandong Jianzhu University, Jinan 250101, China \\ ${ }^{3}$ Internet of Things and Smart City Innovation Platform, Zhuhai Fudan Innovation Institute, Zhuhai 518057, China
}

Correspondence should be addressed to Baozhu Li; tiger_1984@yeah.net

Received 25 November 2021; Accepted 24 January 2022; Published 12 February 2022

Academic Editor: Libo Gao

Copyright (c) 2022 Dandan Pang et al. This is an open access article distributed under the Creative Commons Attribution License, which permits unrestricted use, distribution, and reproduction in any medium, provided the original work is properly cited.

The defects are usually generated during the structural materials subjected to external loads. Elucidating the position distribution of defects using acoustic emission (AE) technique provides the basis for investigating the failure mechanism and prevention of materials and estimating the location of the potentially dangerous sources. However, the location accuracy is heavily affected by both limitation of localization area and reliance on the premeasured wave velocity. Here, we propose a novel AE source localization approach based on generalized areal coordinates and a machine learning algorithmic model. A total of $14641 \mathrm{AE}$ source location simulation cases are carried out to validate the proposed method. The simulation results indicate that even under various measurement error conditions the AE sources could be effectively located. Moreover, the feasibility of the proposed approach is experimentally verified on the AE source localization system. The experiment results show that the mean localization error of $3.64 \mathrm{~mm}$ and the standard deviation of $2.61 \mathrm{~mm}$ are obtained, which are $67.55 \%$ and $75.46 \%$ higher than those of the traditional method.

\section{Introduction}

The defect source location prediction based on acoustic emission (AE) technique is to determine the specific locations of the defects by analyzing the $\mathrm{AE}$ signals generated by structural materials with developing cracks. In recent years, numerous efforts have been dedicated to AE source localization area. The existing researches can be mainly divided into four classes: geometric location method [1-4], sensor array location method [5-8], point search method [9-13], and artificial intelligence algorithm [14-17].

The geometric location method is simple and easy to implement, but it is limited to some parameters, such as sensor coordinates, signal propagation time, and wave speed. The more parameters required, the more errors may be introduced. Also, this method often has multiple solutions [18]. The sensor array location method can locate the damage source according to the sensor array layout and only needs less information to calculate the AE source location. However, this method needs to meet certain positioning prerequisites (for instance, the literature [8] requires a longer distance from the acoustic emission source than the distance between the two sensors in the array) or has a larger positioning error in some monitoring ranges. The point search localization method has a relatively intuitive positioning effect, but this method requires a large amount of preexperimental work and does not fully consider the influence of the dispersion effect and the anisotropy of the AE signal speed on the positioning error [19]. To derive from the methods mentioned above, we can see that the accuracy of AE source localization is dependent on the precision of $\mathrm{AE}$ signal speed measurement. Therefore, it is necessary to get rid of the dependency on the AE signal speed measurement to improve the localization accuracy.

Recently, several localization methods without AE signal speed measurement have been reported. Dong et al. [20, 21] developed a velocity-free MS/AE source location method in complex three-dimensional hole-containing structures. Fang et al. [22] gave a location algorithm with unknown velocity based on INGLADA algorithm. Zhou et al. $[23,24]$ provided 
an algebraic solution for AE source localization without premeasuring wave velocity. With the development of artificial intelligence, machine learning methods have also been introduced into AE source localization area. Ebrahimkhanlou and Salamone [25] introduced two deep learning approaches to localize AE sources within metallic plates, which utilized the reflection and reverberation patterns of $\mathrm{AE}$ waveforms as well as their dispersive and multimodal characteristics to localize AE sources. Suwansin and Phasukkit [26] proposed deep learning-based $\mathrm{AE}$ scheme for localization of cracks in train rails. However, all these methods are applied to $\mathrm{AE}$ source localization in zone. Moreover, since the input of the deep learning network is the whole AE waveform, these methods often require significant computational resources, which leads to a low-efficient and complex positioning process [27]. To address the above problems, Liu et al. [28] developed a new AE source location method, which combined the generalized regression neural network and the TDM localization method to further improve the localization accuracy. However, despite these localization methods have taken into account to alleviate the influence of the wave velocity, there are still several other sources of measurement error (i.e., instrumentation noise, temperature changes, and systematic errors), which can affect the accuracy of these methods [29, 30]. Moreover, these algorithms commonly investigate the $\mathrm{AE}$ source that occur inside the area surrounding by sensors; very few studies have been implemented outside the area surrounding by sensors.

To further enhance the localization accuracy and improve previous studies, we developed a novel areal coordinate and machine learning-based AE source localization method that can not only realize wave velocity-free localization and consider measurement error disturbances but also avoid limitation of localization area. In order to validate the proposed method, the numerical simulations of 14641 different AE source location cases were conducted, and to compare its performance with the conventional algorithm. Besides the numerical simulation validation, AE signal datasets were prepared for detection and localization tasks to train the proposed model; then, the feasibility of the proposed method was experimentally verified on the test datasets. The results were used to test the developed method against our previous study.

\section{Localization Approach}

In this research study, an improved areal coordinate-based AE source localization method is adopted to estimate the coordinates of AE sources. This algorithm starts with determining the signed areal coordinates on triangles [31]. Consider a triangle area surrounded by three AE sensors $i, j$, and $k$, an $\mathrm{AE}$ source $S$ occurs in this area as shown in Figure 1.

2.1. The Principle of Generalized Areal Coordinate-Based AE Source Localization Method. In order to improve the location accuracy and avoid the outliers near the boundary, a generalized areal coordinate-based source localization method which is developed based on the traditional areal coordinate-based source localization method is used to locate the $\mathrm{AE}$ source.

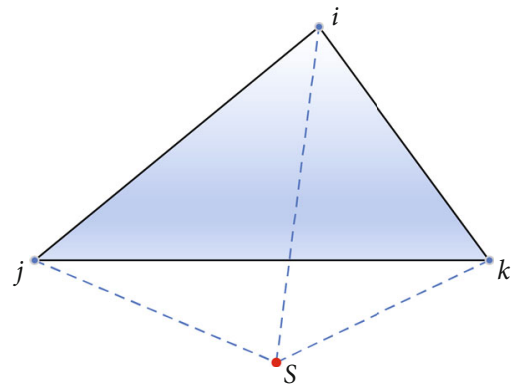

Figure 1: An example of AE source $S$ and three sensors $i, j$, and $k$ for localization approach.

The most obvious difference between the two methods is the number of sensors. There are more than three AE sensors in the generalized areal coordinate-based source localization method. The strategy of updating the Euclidean coordinate of AE source $p_{s}$ can be expressed as

$$
\begin{gathered}
p_{s}=\sum_{i=1}^{n} a_{s i} p_{i}, \\
\sum_{i=1}^{n} a_{s i}=1,
\end{gathered}
$$

where $p_{1}, p_{2}, \ldots, p_{n}$ are the Euclidean coordinates of the $n$ AE sensors and $a_{s 1}, a_{s 2}, \ldots, a_{s n}$ are the areal coordinates of the $n \mathrm{AE}$ sensors. Choose three of the $n \mathrm{AE}$ sensors randomly, until there is no combination of three sensors that have not been selected before. The $m$ th areal coordinate of AE source $S$ with respect to sensors $i, j$, and $k$ is $\left\{a_{s i}^{(m)}, a_{s j}^{(m)}, a_{s k}^{(m)}\right\}$, where $a_{s i}^{(m)}, a_{s j}^{(m)}$, and $a_{s k}^{(m)}$ can be calculated as

$$
\begin{aligned}
& a_{s i}^{(m)}=\frac{S_{\Delta s j k}}{S_{\Delta i j k}}, \\
& a_{s j}^{(m)}=\frac{S_{\Delta s k i}}{S_{\Delta i j k}}, \\
& a_{s k}^{(m)}=\frac{S_{\Delta s i j}}{S_{\Delta i j k}},
\end{aligned}
$$

where $S_{\Delta s j k}, S_{\Delta s k i}, S_{\Delta s i j}$, and $S_{\Delta i j k}$ are the sign areas of triangles, which can be computed through Cayley-Menger determinant with the distance measurements among AE source $S$ and sensors $i, j, k, d_{i j}, d_{i k}, d_{k j}, d_{s i}, d_{s j}$, and $d_{s k}$.

The generalized areal coordinate of AE source $S$ with respect to $n \mathrm{AE}$ sensors is defined as

$$
a_{s i}=\left(\frac{1}{m}\right) \sum_{r=1}^{m} a_{s i}^{(m)}, \quad i \in n .
$$

According to Equation (3), we can see that $a_{s i}$ is the average measurement value of areal coordinate. Therefore, the obtained areal coordinate $a_{s i}$ can achieve a higher accuracy and avoid the outliers. 
2.2. The Principle of Improved AE Source Localization Method. According to the above analysis, we find that the key factor affecting the localization accuracy is the accuracy of the measurement distances from the AE source $S$ to the sensors. It is easy to be affected by the propagation speed and arrival time of $\mathrm{AE}$ wave, and it is inevitable to have a certain measurement error. Based on this, we propose a new location method without measuring $\mathrm{AE}$ wave velocity and considering measurement error. We use the characteristics of $\mathrm{AE}$ wave and artificial neural network (ANN) technology to deduce the distance between AE source and sensor without measuring wave velocity (see Section 4.1 for specific implementation principle). On this basis, we also improve the positioning algorithm based on increasing the measurement error factor to reduce the impact of unavoidable measurement errors on the accuracy of the positioning algorithm.

Specifically, in the measurement errors existed condition, the distance measurements among AE source $S$ and sensors $i, j$, and $k$ are modeled as follows:

$$
\begin{aligned}
& \widehat{d}_{s i}=d_{s i}+e_{i}, \\
& \widehat{d}_{s j}=d_{s j}+e_{j}, \\
& \widehat{d}_{s k}=d_{s k}+e_{k},
\end{aligned}
$$

where $e_{i}, e_{j}$, and $e_{k}$ are the measurement errors. And the measurement error obeys Gaussian, Uniform, or Exponential distribution [32]. The distribution and parameters of the errors are different in various environments and measurement methods.

Due to the effect of measurement errors, the original calculation method will not work when solving the values of areal coordinate $\left\{a_{s i}^{(m)}, a_{s j}^{(m)}, a_{s k}^{(m)}\right\}$. Therefore, it is necessary to modify the original equations or constraints so that they can still correctly work with measurement errors. Solving the values of $\left\{a_{s i}^{(m)}, a_{s j}^{(m)}, a_{s k}^{(m)}\right\}$ can be divided into two steps. First, obtain the unsigned values of $\left\{a_{s i}^{(m)}, a_{s j}^{(m)}, a_{s k}^{(m)}\right\}$, and then determine the signs of $\left\{a_{s i}^{(m)}, a_{s j}^{(m)}, a_{s k}^{(m)}\right\}$. More specifically, we calculate the unsigned areal coordinates $\left|a_{s i}^{(m)}\right|$, $\left|a_{s j}^{(m)}\right|$, and $\left|a_{s k}^{(m)}\right|$. Due to the linear relationship, when the triangle area is close to 0 , the corresponding areal coordinate is also close to 0 . The signed area value of triangles $S_{\Delta s j k}$ may be a small negative number because of the existence of measurement error. The absolute value of $S_{\Delta s j k}$ is firstly used to reduce the error as much as possible

$$
\left|S_{\Delta s j k}\right|=\sqrt{\left|-\frac{1}{16}\right| \begin{array}{cccc}
0 & 1 & 1 & 1 \\
1 & 0 & \hat{d}_{s j}^{2} & \hat{d}_{s k}^{2} \\
1 & \hat{d}_{j s}^{2} & 0 & \hat{d}_{j k}^{2} \\
1 & \hat{d}_{k s}^{2} & \hat{d}_{k j}^{2} & 0
\end{array} \mid} .
$$

After obtaining the unsigned values of $\left|a_{s i}^{(m)}\right|,\left|a_{s j}^{(m)}\right|$, and $\left|a_{s k}^{(m)}\right|$, the next step is to judge and solve the sign patterns $\left(\sigma_{s i}, \sigma_{s j}, \sigma_{s k}\right)$. Equation (6) is modified as

$$
1-\Delta_{1} \leq \sum_{i=1}^{n} a_{s i} \leq 1+\Delta_{1},
$$

where $\Delta_{1}$ is a small positive number, which changes depending on the measurement error. When $n$ is 3 (such as the model shown in Figure 1), this equation can be rewritten as

$$
1-\Delta_{1} \leq \sigma_{s i}\left|a_{s i}\right|+\sigma_{s j}\left|a_{s j}\right|+\sigma_{s k}\left|a_{s k}\right| \leq 1+\Delta_{1} \text {, }
$$

where $\sigma_{s i}, \sigma_{s j}$, and $\sigma_{s k}$ are the signs of the coordinates and take values of either -1 or 1 . Then, 7 possible combinations of $\left(\sigma_{s i}, \sigma_{s j}, \sigma_{s k}\right)$ are substituted into Equation (7), and the corresponding number of solutions is solved.

After the sign determination, the localization of the AE source $S$ is accomplished in the measurement error existed condition. The procedure of improved AE source localization method is illustrated in Figure 2.

\section{Simulation Validation}

To validate the performance of the proposed method in the previous section, numerical simulations are conducted to estimate the coordinate of the AE source. A total of 14641 different AE source locations are simulated on the plate, as shown in Figure 3. The interval is $5 \mathrm{~mm}$ between the two adjacent simulated AE source locations. The dimension of the simulated area is $600 \mathrm{~mm} \times 600 \mathrm{~mm}$.

3.1. The Traditional AE Source Localization Method without Measurement Error. When there is no measurement error, the obtained measurement distances $d_{s j}, d_{s k}$, and $d_{s i}$ are real values; the positioning of the $\mathrm{AE}$ source is completed using the traditional areal coordinate method based on the sensor distances $d_{i j}, d_{j k}$, and $d_{k i}$ and the measurement distances $d_{s j}$, $d_{s k}$, and $d_{s i}$.

For the case of no measurement error, the traditional areal coordinate-based AE source localization method is performed. Numerical simulations are conducted using the MATLAB software; the coordinates of the three AE sensors are set at $(-80 \mathrm{~mm}, 0 \mathrm{~mm}),(80 \mathrm{~mm}, 0 \mathrm{~mm})$, and $(0 \mathrm{~mm}$, $80 \mathrm{~mm}$ ) for simulation. As shown in Figure 3, the simulated abscissa and ordinate localization errors are less than 1.55 and $1.85 e-12 \mathrm{~mm}$, the average abscissa and ordinate localization errors are 0.004 and $3.34 e-14 \mathrm{~mm}$, and the standard deviation of the abscissa and ordinate localization errors is 0.06 and $5.61 e-14 \mathrm{~mm}$. Because the average grid size for simulation is $5 \mathrm{~mm}$, these localization results can be considered reasonable. While the above results will be changed if there are measurement errors, so it is necessary to make improvements to adapt to the measurement error existed situation.

3.2. The Traditional AE Source Localization Method with Measurement Error. A total of 30 different AE source localization experiments of applying random noise were simulated by using the traditional areal coordinate method. The performance 


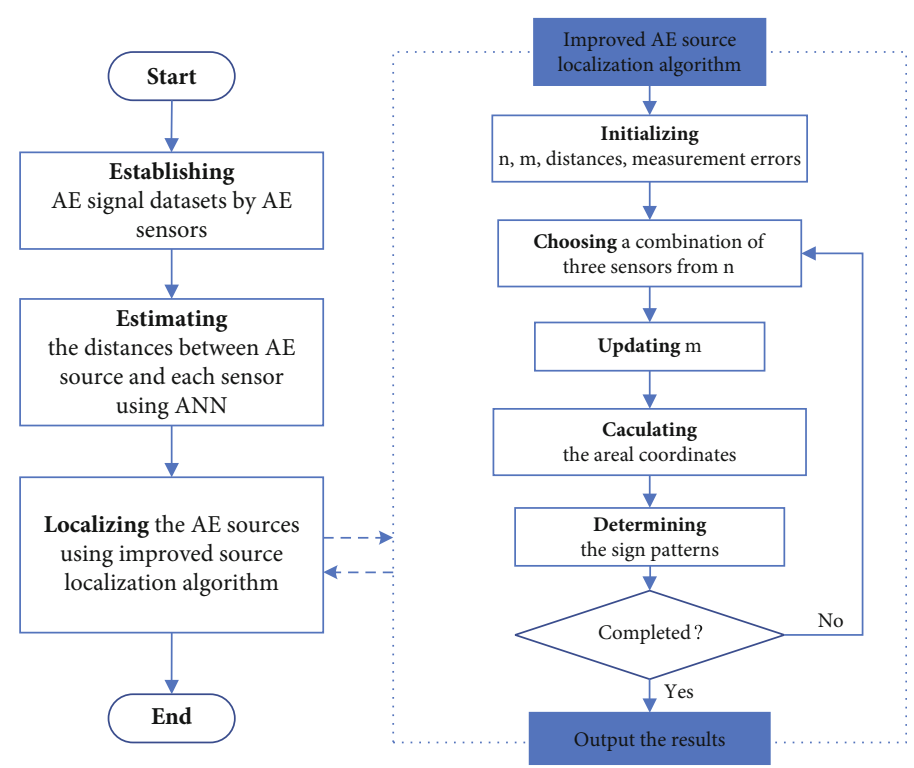

FIgURE 2: Procedure of improved AE source localization.

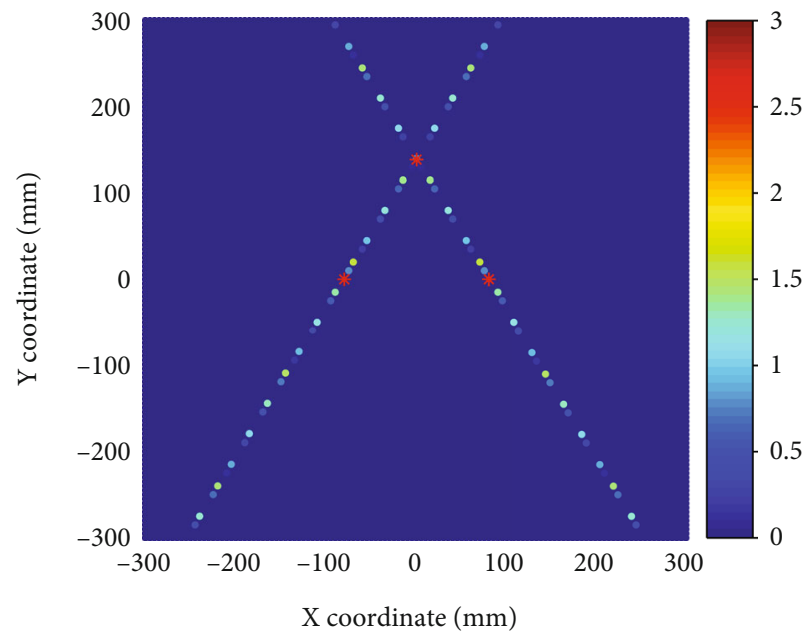

(a)

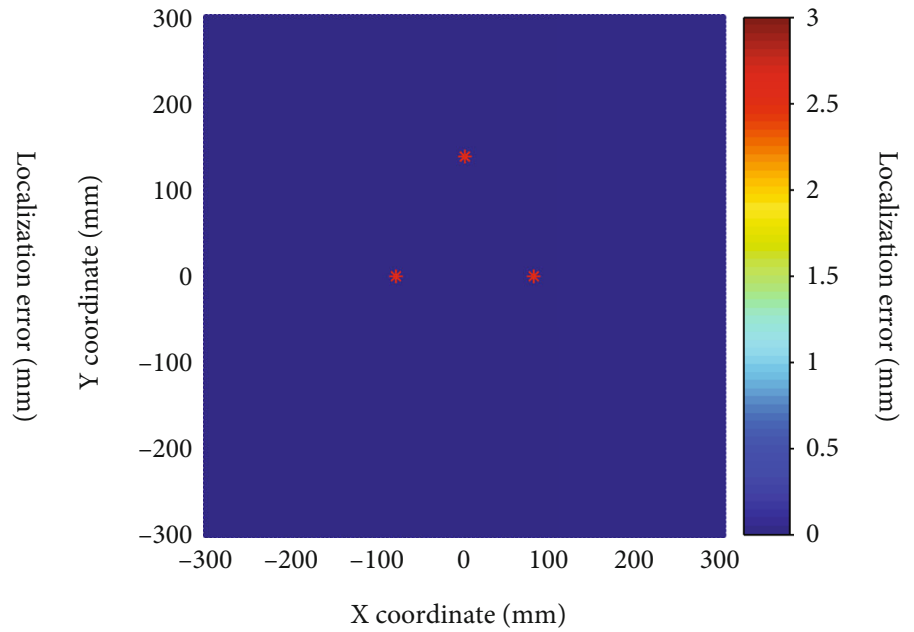

(b)

FIGURE 3: The simulation results of the traditional areal coordinate-based AE source localization method without measurement error: (a) the abscissa localization error; (b) the ordinate localization error.

of traditional method is investigated when the measurement error obeys the Uniform distribution $\left(e_{i} \sim U(-1,1)\right)$ and the Gaussian distribution $\left(e_{i} \sim N\left(0,1^{2}\right)\right)$, as shown in Figure 4 . If the measurement error obeys the Uniform distribution $\left(e_{i} \sim U(-1,1)\right)$, the simulated abscissa and ordinate localization errors are less than 602.19 and $603.34 \mathrm{~mm}$, the average abscissa and ordinate localization errors are 4.43 and $4.15 \mathrm{~mm}$, and the standard deviation of the abscissa and ordinate localization errors is less than 29.89 and $37.16 \mathrm{~mm}$. If the measurement error obeys the Gaussian distribution $\left(e_{i} \sim N\left(0,1^{2}\right)\right)$, the simulated abscissa and ordinate localization errors are less than 604.54 and $609.12 \mathrm{~mm}$, the average abscissa and ordinate localization errors are 7.34 and $7.06 \mathrm{~mm}$, and the standard deviation of the abscissa and ordinate localization errors is less than 39.36 and $49.00 \mathrm{~mm}$.
The simulation results indicate that the AE source localization algorithm has good adaptability and robustness; however, there are still some cases where large error points will occur, especially when the AE source lies on the line parallel to one side of the triangle of three sensors. Thus, in order to enhance the localization accuracy, an improved AE source localization method is proposed.

3.3. The Improved AE Source Localization Method with Measurement Error. After introducing the measurement error, the problem that has not been solved is the misjudgment of the sign pattern, which will cause localization errors. In order to solve this problem, we propose an improved localization method, which has been introduced in Section 2; taking four sensor nodes as an example, any three of the 


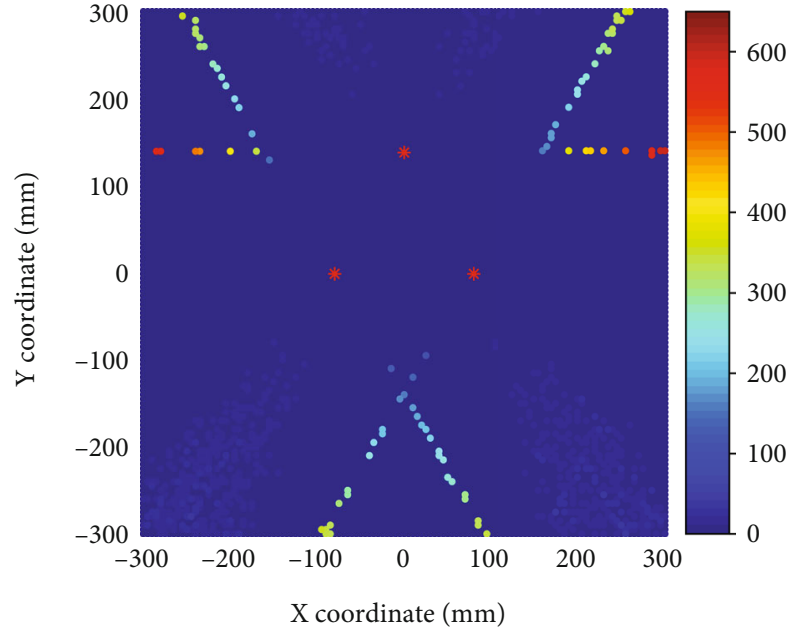

(a)

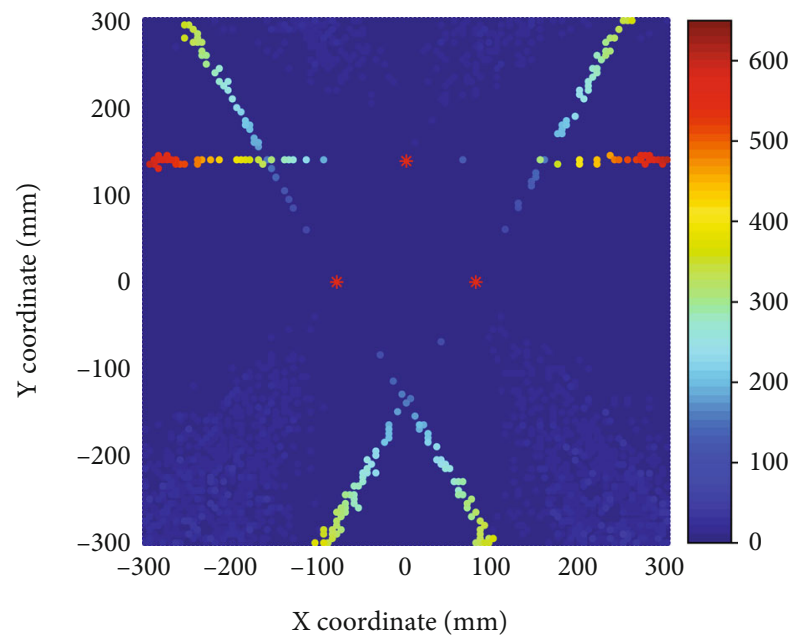

(c)

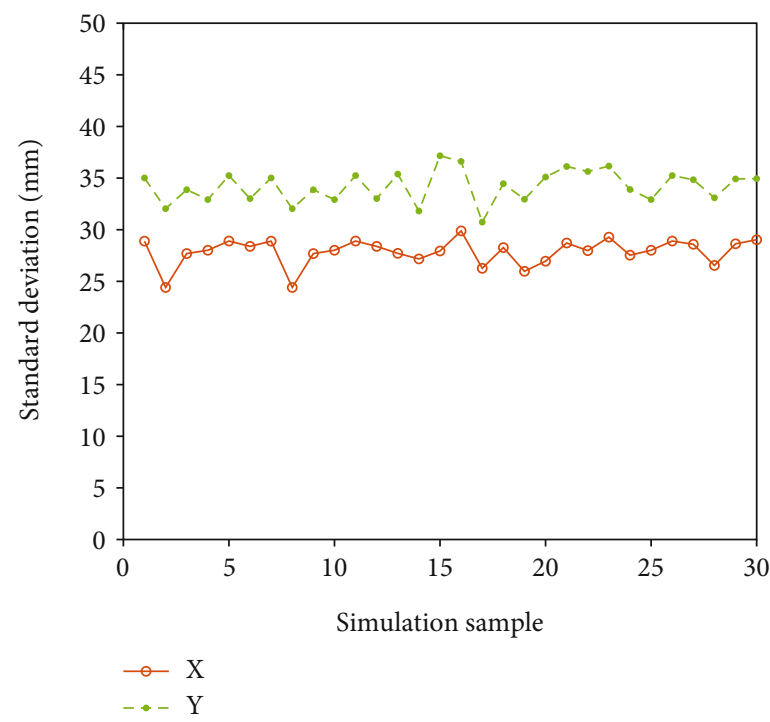

(e)

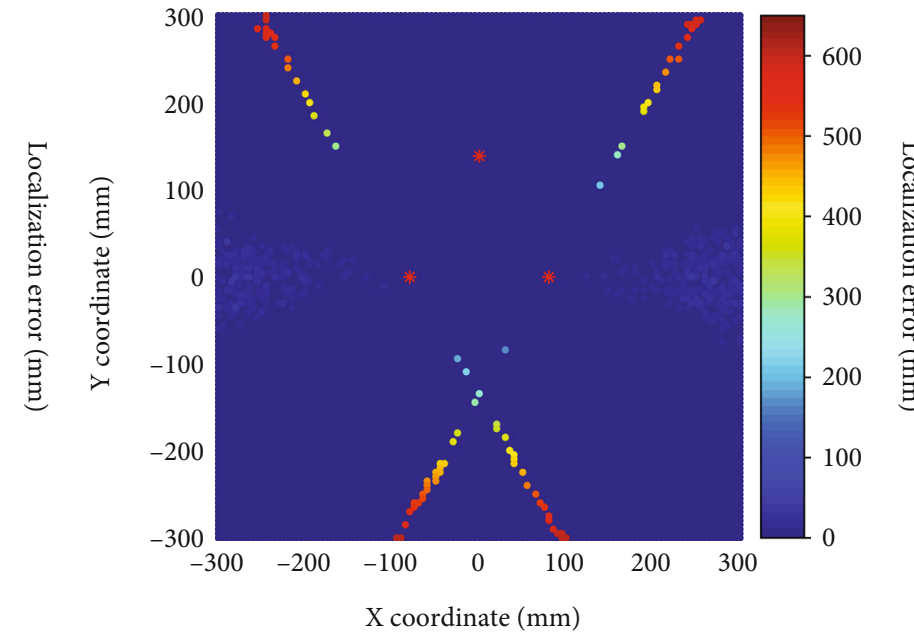

(b)

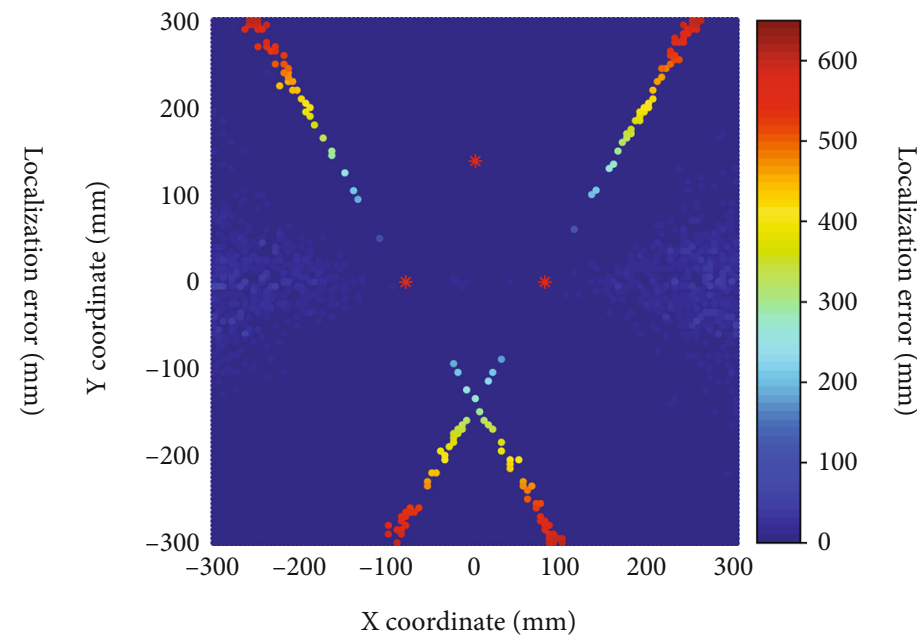

(d)

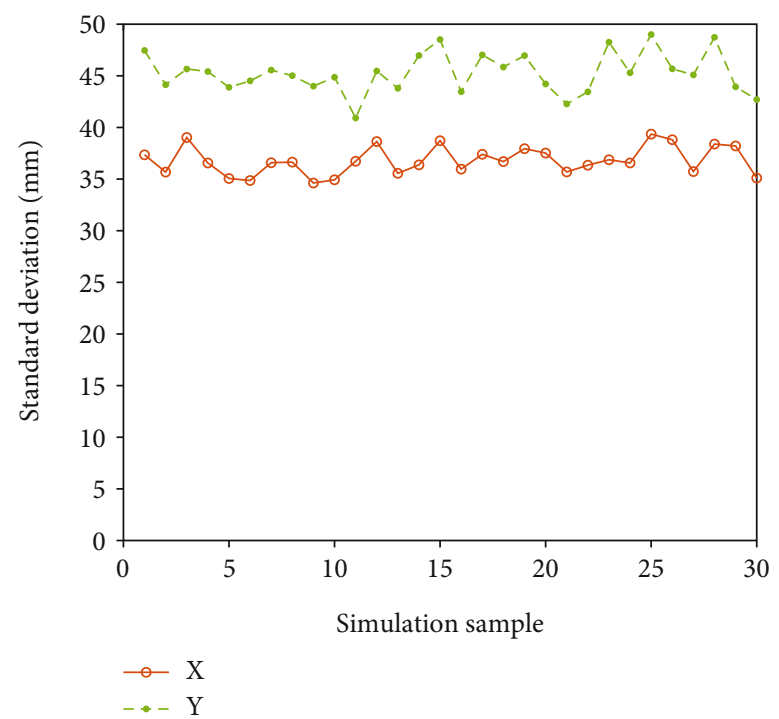

(f)

FIgURE 4: The simulation results of the traditional areal coordinate-based AE source localization method with measurement error: (a, b, e) localization errors and standard deviation when the measurement error obeys the Uniform distribution; (c, d, f) localization errors and standard deviation when the measurement error obeys the Gaussian distribution. 


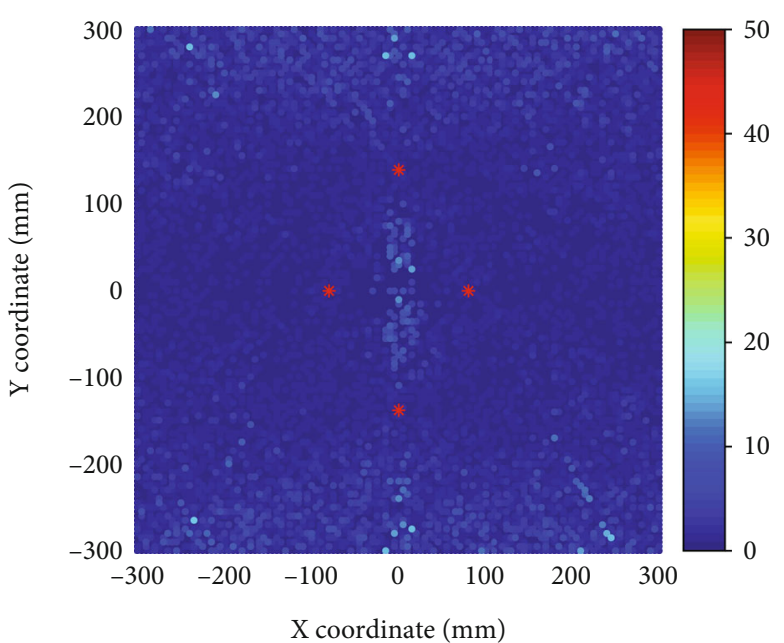

(a)

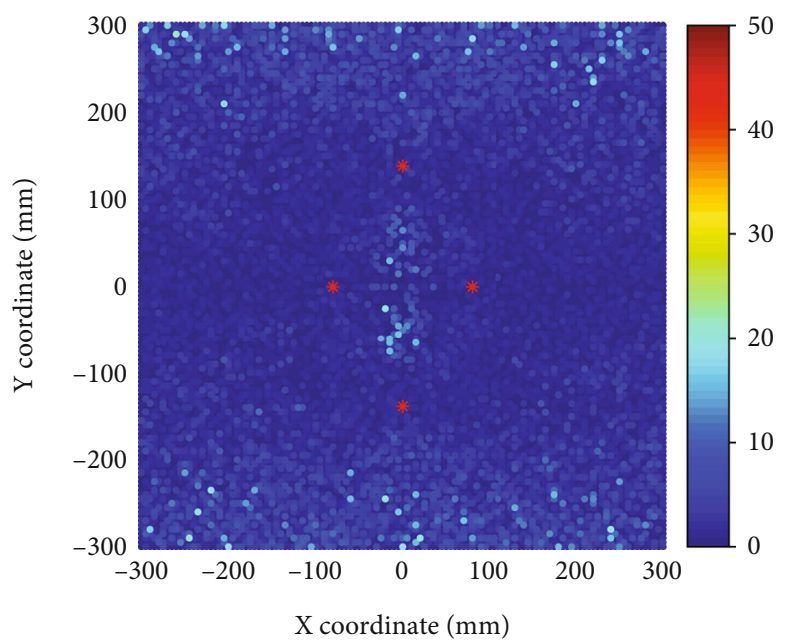

(c)

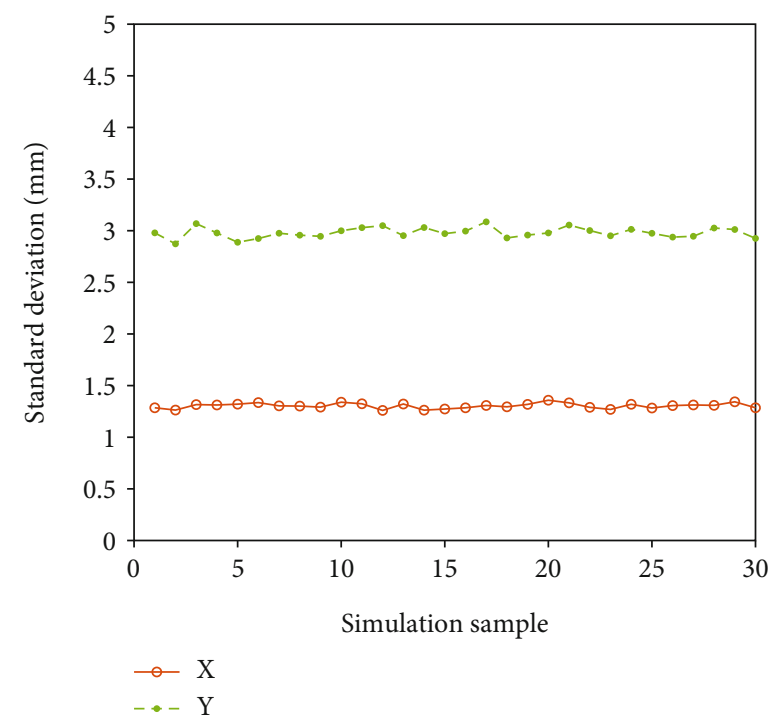

(e)

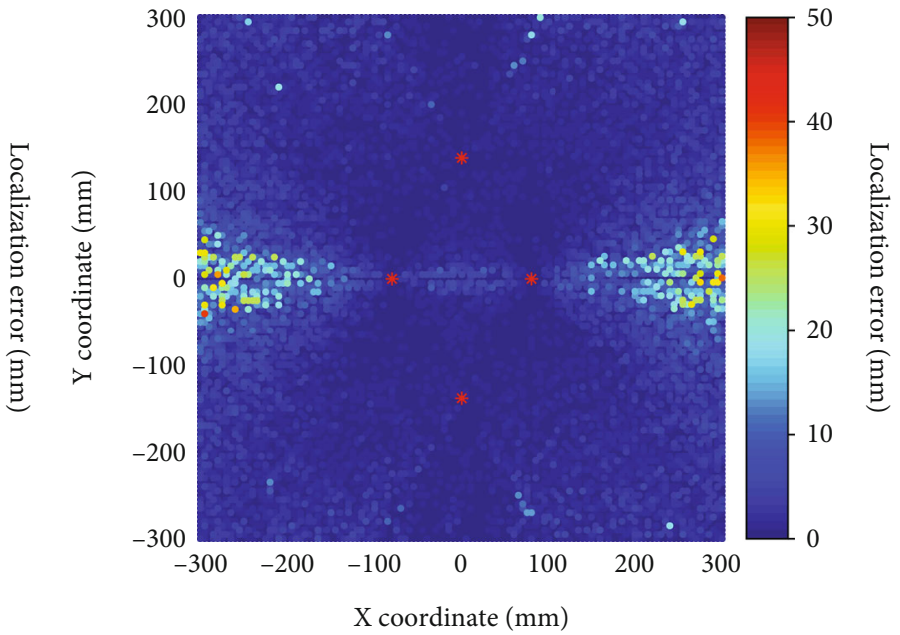

(b)

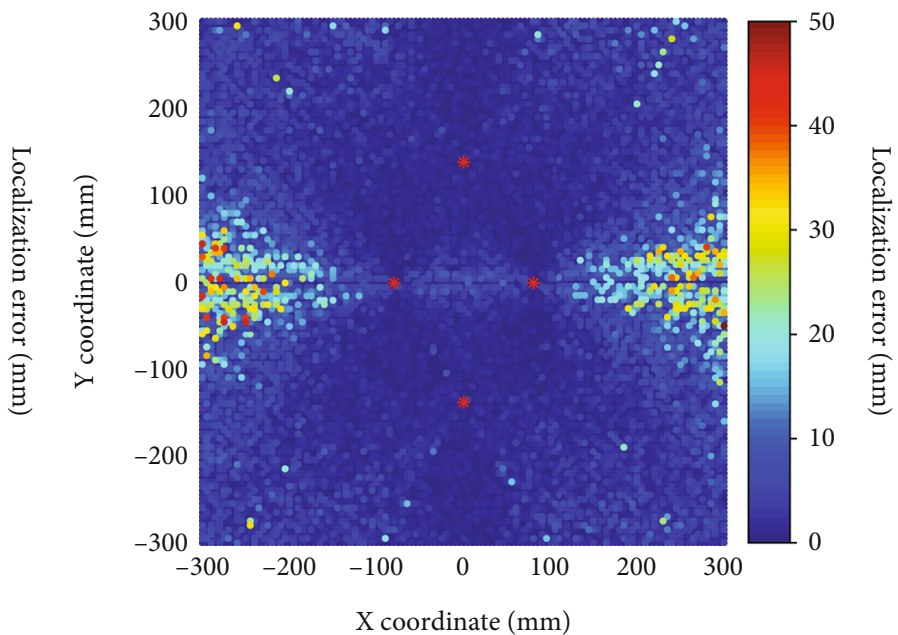

(d)

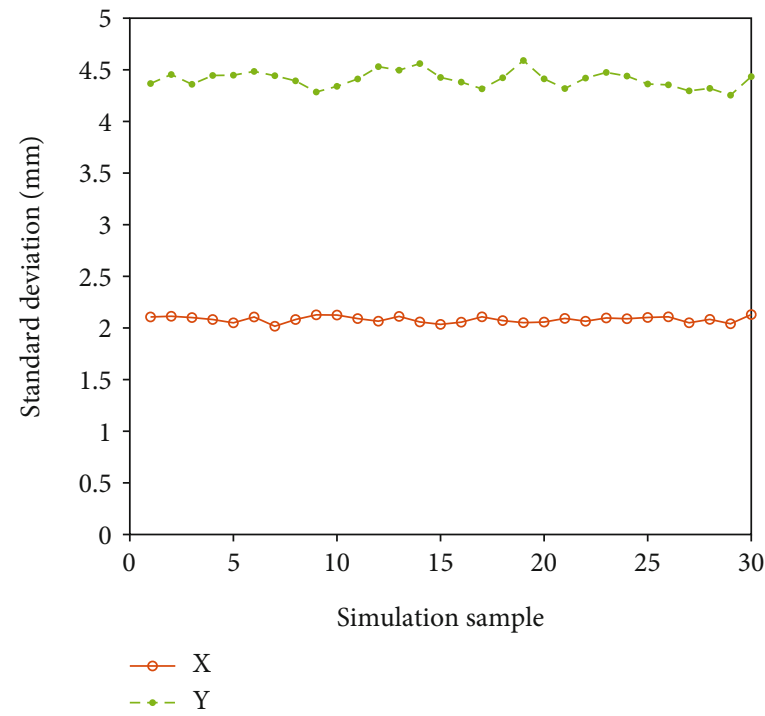

(f)

FIGURE 5: The simulation results of the generalized areal coordinate-based AE source localization method with measurement error: (a, b, e) localization errors and standard deviation when the measurement error obeys the Uniform distribution; (c, d, f) localization errors and standard deviation when the measurement error obeys the Gaussian distribution. 


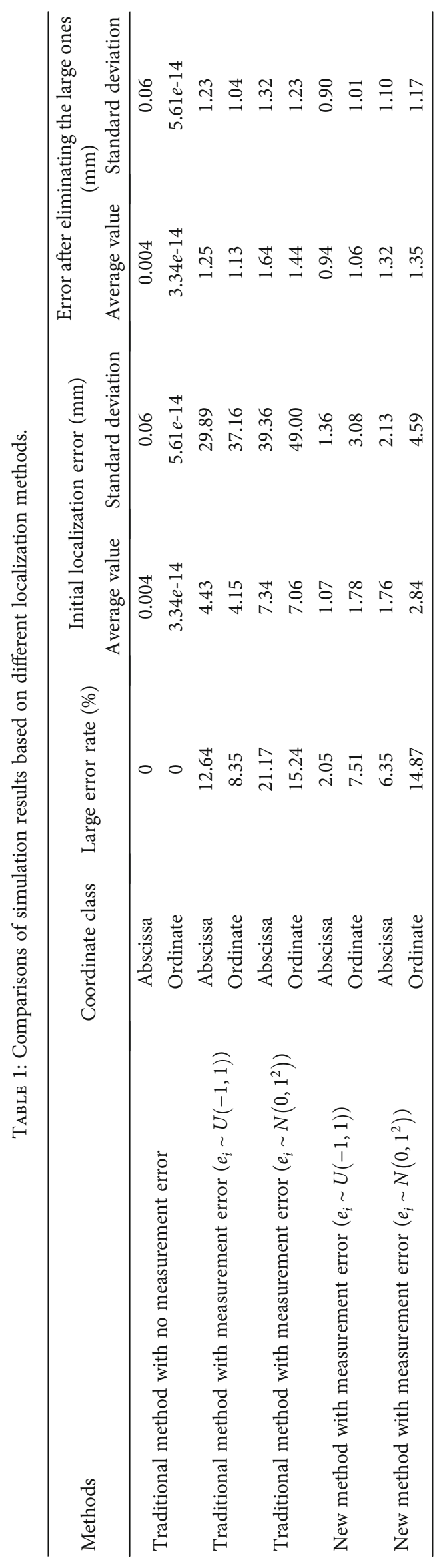


four nodes can form a triangle, so the respective localization results of the AE source $S$ can be obtained based on the four AE sensors. From these obtained four sets of localization coordinates, one set of relatively outlier data can be removed, and the remaining three sets of data are averaged to compute a new localization result.

Specifically, the improved AE source localization method with measurement error is performed into the following steps.

Step 1. There are four AE sensors, the coordinates of the three AE sensors are set at $(-80 \mathrm{~mm}, 0 \mathrm{~mm}),(80 \mathrm{~mm}, 0 \mathrm{~mm}),(0 \mathrm{~mm}$, $-80 \sqrt{3} \mathrm{~mm})$, and $(0 \mathrm{~mm}, 80 \sqrt{3} \mathrm{~mm})$ for simulation, selecting three out of the four nodes in turn as a group and performing AE source localization according to the method in Part B to obtain the AE source coordinates $\left(x_{1}, y_{1}\right)$.

Step 2. The first step is repeated in turn, and the other source coordinates $\left(x_{2}, y_{2}\right),\left(x_{3}, y_{3}\right)$, and $\left(x_{4}, y_{4}\right)$ are all calculated by taken possible combinations of the sensor groups.

Step 3. The obtained four AE source coordinates are compared. The one farthest from the other three coordinates is deleted from the four coordinates, leaving three coordinates.

Step 4. Then, the point farthest from the remaining two points is deleted from the three coordinates, leaving only two coordinates. The remaining two coordinates are averaged to obtain the final AE source coordinates.

The performance of proposed method is investigated when the measurement error obeys the Uniform distribution $\left(e_{i} \sim U(-1,1)\right)$ and the Gaussian distribution $\left(e_{i} \sim N\left(0,1^{2}\right)\right)$, as shown in Figure 5. If the measurement error obeys the Uniform distribution $\left(e_{i} \sim U(-1,1)\right)$, the simulated abscissa and ordinate localization errors are less than 25 and $42.31 \mathrm{~mm}$, the average abscissa and ordinate localization errors are 1.07 and $1.78 \mathrm{~mm}$, and the standard deviation of the abscissa and ordinate localization errors is less than 1.36 and $3.08 \mathrm{~mm}$. If the measurement error obeys the Gaussian distribution $\left(e_{i} \sim N\left(0,1^{2}\right)\right)$, the simulated abscissa and ordinate localization errors are less than 30 and $68.34 \mathrm{~mm}$, the average abscissa and ordinate localization errors are 1.76 and $2.84 \mathrm{~mm}$, and the standard deviation of the abscissa and ordinate localization errors is less than 2.13 and $4.59 \mathrm{~mm}$.

Considering the average grid size of simulation is $5 \mathrm{~mm}$, we set the localization error which is greater than or equal to $5 \mathrm{~mm}$ as the large error. Compute and compare the large error rates of simulation results based on different localization methods (Table 1). Compared with the other four cases, traditional method with measurement error that obeys the Gaussian distribution has the highest large error rate. Besides, new method with measurement error that obeys the Uniform distribution has the lowest large error rate among the methods with measurement error. The average value and standard deviation of the initial simulation results and the results after eliminating the large errors are also compared in Table 1. It can be seen that the values of all the methods with measure-

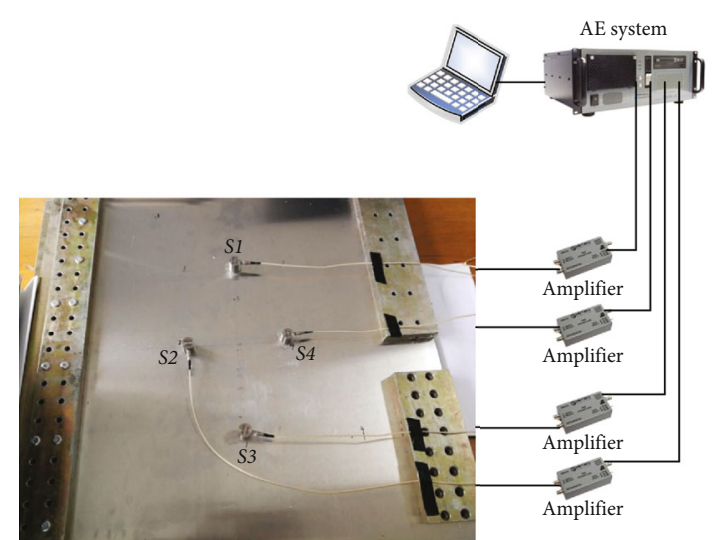

FIgURE 6: The experimental setup.

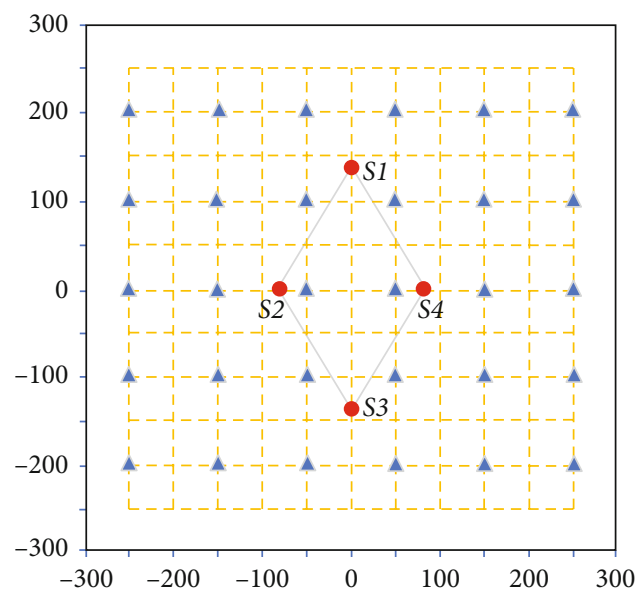

Figure 7: AE source locations of the dataset ( $\mathbf{\Delta}$ : test sets, unit: $\mathrm{mm}$ ).

ment error are reduced. However, the decrease in the value of traditional methods is obvious, which means the traditional methods are more vulnerable to measurement errors, and the new methods can achieve competitive performance.

\section{Experiments}

In this section, the performance of the introduced generalized areal coordinate method for monitored structure $\mathrm{AE}$ source localization based on the PZT sensors was experimentally validated. The experimental set up is elaborated in Section 4.1, and the experimental results and analysis are given in Section 4.2.

4.1. Experimental Setup. The experimental setup was used to evaluate the effectiveness of the proposed AE source localization approach, as shown in Figure 6. The experiment system was mainly composed of AE data acquisition equipment, aluminum alloy plate, four PZT sensors, and preamplifiers. The sampling frequency was up to $1 \mathrm{MHz}$. The specimen was a sheet metal (material: 6061-T6 aluminum). The dimensions of the specimen were as follows: $600 \mathrm{~mm} \times 600 \mathrm{~mm} \times 3 \mathrm{~mm}$. To collect AE signals, four AE sensors were mounted with Vaseline on the surface of the plate. Based on the previous studies, the sensors were attached at the coordinates of $S 1$ 


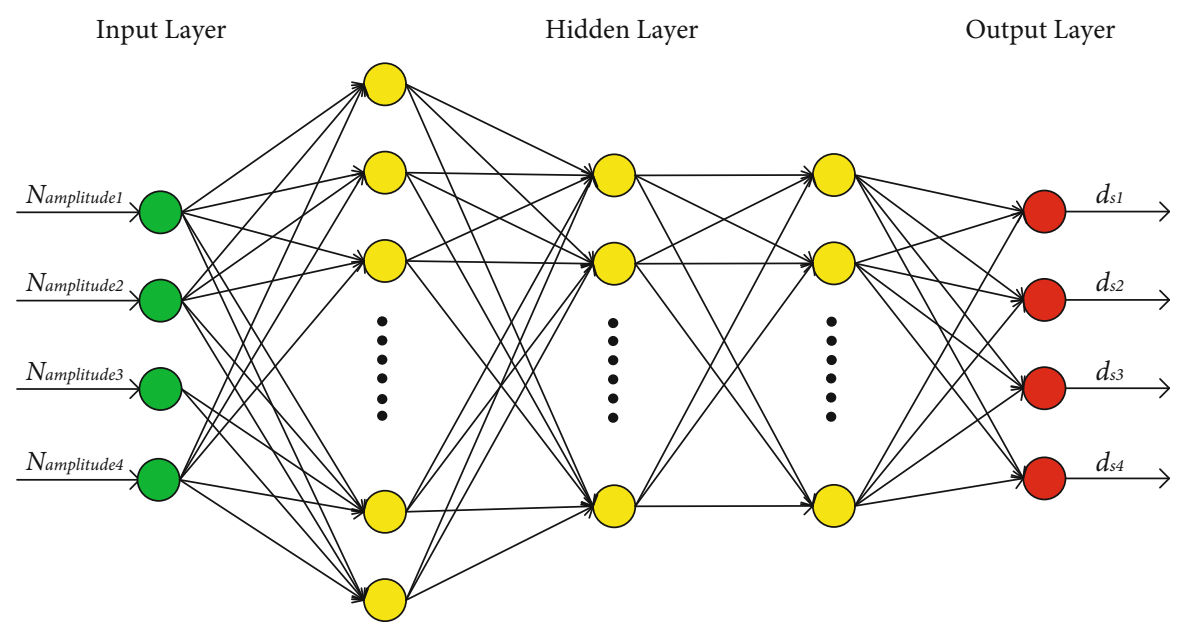

FIGURE 8: The proposed neural network structure.

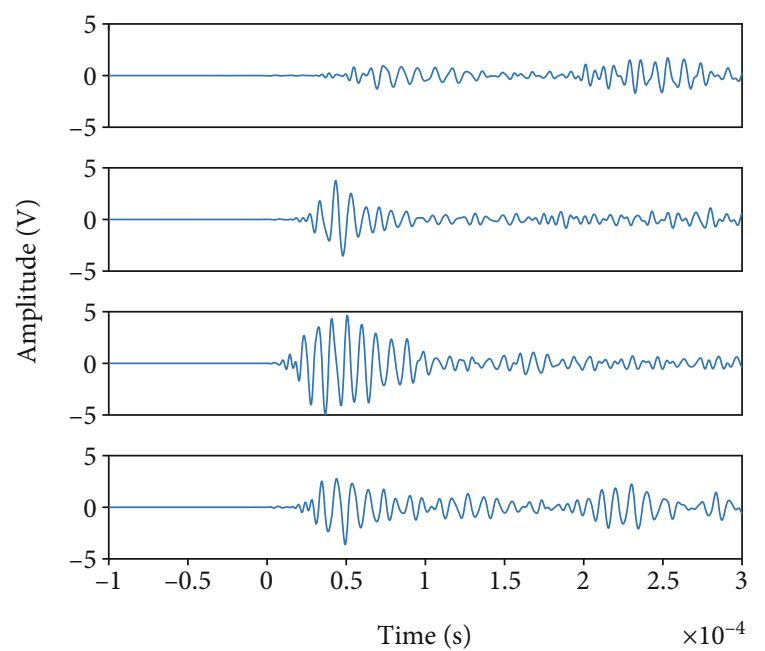

Figure 9: One example of the detected AE signals.

(0mm, $80 \sqrt{3} \mathrm{~mm}), \mathrm{S} 2(-80 \mathrm{~mm}, 0 \mathrm{~mm}), \mathrm{S} 3(0 \mathrm{~mm},-80 \sqrt{3}$ $\mathrm{mm})$, and $S 4(80 \mathrm{~mm}, 0 \mathrm{~mm})$, respectively, relative to the center point of the plate. AE sources were generated by HsuNielsen pencil lead breaks. As shown in Figure 7, the experimental area was $50 \mathrm{~mm}$ from the edge of the plate, and the grid size for AE tests was $50 \mathrm{~mm}$. All the datasets were located at the intersection of the dotted line plotted in this figure, in which 61 points, 30 points, and 30 points were considered as training, validation, and test sets, respectively. In each point, 3 Hsu-Nielsen pencil lead break tests were simulated.

Before data collection, the AE signals were preamplified by $40 \mathrm{~dB}$ and filtered to the frequency range of $100 \mathrm{kHz}-$ $200 \mathrm{kHz}$. The extracted narrowband signals can be obtained after processed by the Shannon wavelet transform and peak detection algorithms. From previous researches [33-35], it was found that apart from TOA, amplitude values of AE signals have shown great potential in AE source localization because of the excellent relationship between the amplitude value of $\mathrm{AE}$ signal and the damage distance from each $\mathrm{AE}$

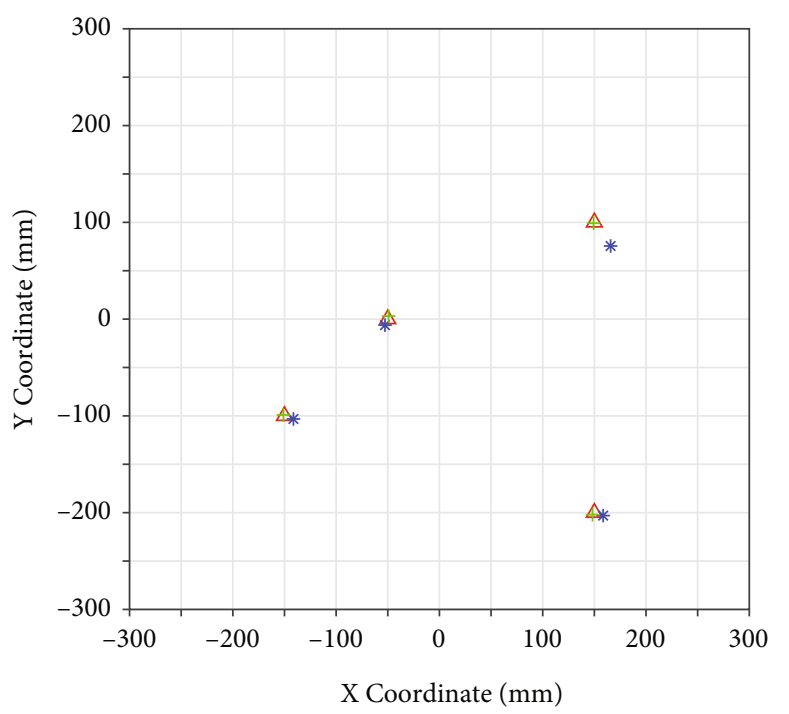

$$
\begin{aligned}
& \triangle \text { Actual } \\
& * \text { Traditional } \\
& + \text { New }
\end{aligned}
$$

FIgURE 10: The exampled localization results.

sensor. In this paper, the amplitude values of AE signals can be obtained according to the extracted narrowband signals. We used the maximum amplitude value of the extracted narrowband signal. Then, the normalized amplitude value of each sensor signal $N_{\text {amplitudei }}$ was computed as below

$$
N_{\text {amplitude } i}=\frac{\left(\text { amplitude }_{i}-\overline{\text { amplitude }}\right)}{\sigma},
$$

where amplitude is the average value of signal amplitudes and $\sigma$ is the standard deviation of signal amplitudes.

Artificial neural network (ANN) which is a useful solution of nonlinear problem was adopted to enhance accuracy of the estimated distances. In this paper, the used neural 
TABLE 2: The AE source location coordinates (unit: $\mathrm{mm}$ ).

\begin{tabular}{lccccccccc}
\hline No. & Actual coordinate & No. & Actual coordinate & No. & Actual coordinate & No. & Actual coordinate & No. & Actual coordinate \\
\hline N-1 & $(-250,200)$ & N-2 & $(-150,200)$ & N-3 & $(-50,200)$ & N-4 & $(50,200)$ & N-5 & $(150,200)$ \\
N-6 & $(250,200)$ & N-7 & $(-250,100)$ & N-8 & $(-150,100)$ & N-9 & $(-50,100)$ & N-10 & $(50,100)$ \\
N-11 & $(150,100)$ & N-12 & $(250,100)$ & N-13 & $(-250,0)$ & N-14 & $(-150,0)$ & N-15 & $(-50,0)$ \\
N-16 & $(50,0)$ & N-17 & $(150,0)$ & N-18 & $(250,0)$ & N-19 & $(-250,-100)$ & N-20 & $(-150,-100)$ \\
N-21 & $(-50,-100)$ & N-22 & $(50,-100)$ & N-23 & $(150,-100)$ & N-24 & $(250,-100)$ & N-25 & $(-250,-200)$ \\
N-26 & $(-150,-200)$ & N-27 & $(-50,-200)$ & N-28 & $(50,-200)$ & N-29 & $(150,-200)$ & N-30 & $(250,-200)$ \\
\hline
\end{tabular}

network is composed of 3 hidden layers with 32,16 and 16 nodes, respectively, as shown in Figure 8. The input data is the normalized amplitude value $N_{\text {amplitudei }}$, and the output data is the distance between each sensor and AE source $d_{s i}$. As shown in Figure 7, there were $363 \mathrm{AE}$ signals in this research. The AE signal datasets were divided into a training dataset (50\%), a validation dataset (25\%), and a testing dataset $(25 \%)$. The number of training dataset is 183 . The validation dataset can provide an unbiased evaluation of a model fit on the training dataset while tuning the model's hyperparameters [36, 37]. This dataset is used for minimizing the overfitting problem. In addition, in order to check the accuracy of training results, 90 test dataset is used. The neural network is improved for fast learning by Quai-Newton method which is an optimization algorithm based on Taylor series expansion. It can be seen that the estimated distances according to the neural network and the true distances coincide well with each other. The distances of AE source from three sensors can be well estimated using the trained weights of the hidden layers. The AE source locations can be predicted through the improved areal coordinate-based $\mathrm{AE}$ source localization method using the estimated distances.

4.2. Results and Analysis. The AE source locations in the test area were calculated using the generalized areal coordinates and ANN-based AE source localization algorithm described above. One example of the detected $\mathrm{AE}$ signals is shown in Figure 9.

The exampled localization results of this experiment are shown in Figure 10. In this illustration, the triangle marker " $\triangle$ " represents the actual coordinate of the AE source, asterisk marker " $*$ " represents the predicted coordinate of the $\mathrm{AE}$ source determined by the traditional areal coordinate-based localization method, and plus marker "+" represents the predicted coordinate of the AE source determined by the new generalized areal coordinate-based localization method. From the experimental results, we can find that the plus markers "+" are always nearer to the triangle markers " $\triangle$ " than the asterisk markers " $*$," which means that compared with the localization results of traditional method, the results of new method have a better performance in coinciding with the actual AE source locations.

The coordinates of $30 \mathrm{AE}$ source locations in test dataset are shown in Table 2. Figure 11 illustrates the results of location errors using the traditional method and our proposed new method. The experimental results show that AE source positions $\mathrm{N}-8, \mathrm{~N}-11, \mathrm{~N}-13, \mathrm{~N}-27$, and $\mathrm{N}-28$ have larger

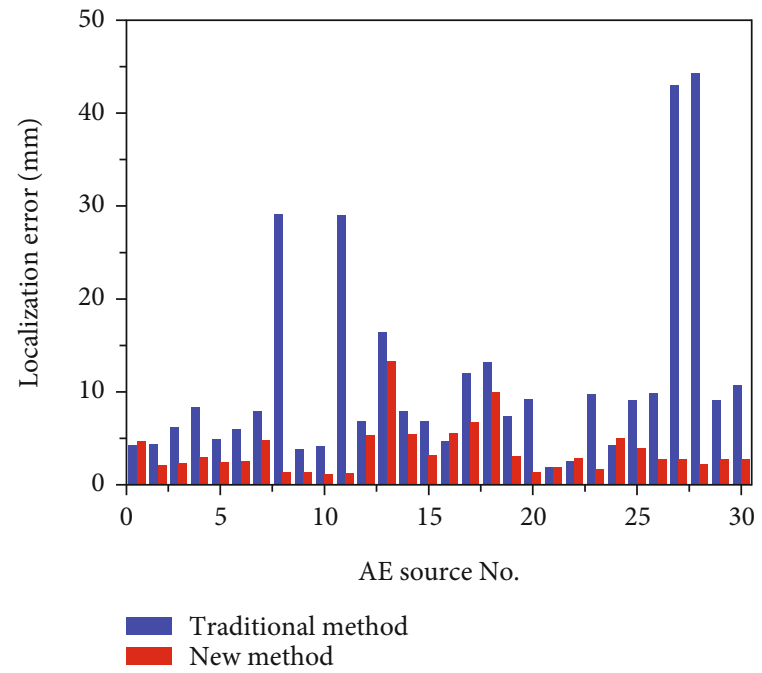

FIgURE 11: The localization errors of 30 AE sources.

errors than other positions ( $>25 \mathrm{~mm}$ ). The larger errors are all resulted by using the traditional method. The maximum localization error obtained using the proposed method is less than $15 \mathrm{~mm}$. Therefore, the proposed new method was found to have higher accuracy in practical AE source localization application. It can be seen that the distribution of localization error is consistent with the estimated error in Section 3. This error seems to be mainly attributed to the locations of these points on the edge of the test area. To expand the coverage area, additional PZT sensors can be used to supplement the limitations. In addition, by adding an appropriate amount of PZT sensors, the positioning performance of this method can be improved.

Figure 12 shows the mean localization errors and standard deviations for the location results of $30 \mathrm{AE}$ sources determined by the two methods. It can be seen that the mean localization error and standard deviation of the proposed method are both smaller than those of the traditional method, which further demonstrates the proposed method holds a more stable and higher location accuracy than the traditional method. In addition, the mean localization error $(3.64 \mathrm{~mm})$ and the standard deviation $(2.61 \mathrm{~mm})$ of the new method are less than the traditional method $(11.23 \mathrm{~mm}$ and $10.67 \mathrm{~mm})$. Therefore, compared with the traditional method, the location accuracy and stability of the proposed method are improved by $67.55 \%$ and $75.46 \%$, respectively. 


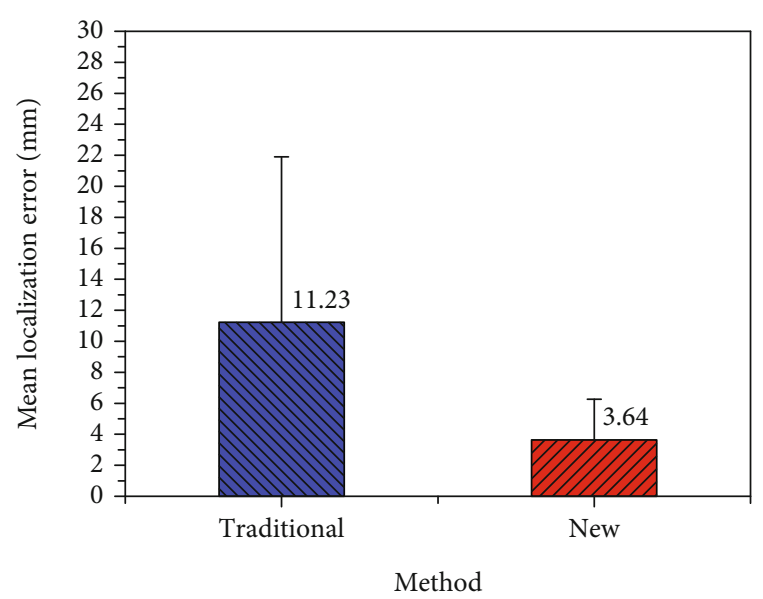

FIGURE 12: The comparison results of the two localization methods.

\section{Conclusion}

This study proposes an improved approach for localization of the potentially defect source in structural material using $\mathrm{AE}$ technique. Compared with the traditional AE source localization algorithms, the proposed method has higher location accuracy and more excellent stability. Besides, considering the measurement error makes the model more robust, which greatly improves the performance of $\mathrm{AE}$ source localization in practical engineering applications. This method also adopts a neural network structure to estimate the damage distances from $\mathrm{AE}$ sensors, which makes the method completely get rid of the reliance on premeasured wave velocity. A total of $14641 \mathrm{AE}$ source location simulation cases are carried out to validate the proposed method. The simulation results indicate that the AE sources could be effectively located, even under various measurement error conditions. Compared with traditional method with no measurement error and the traditional methods with measurement errors, the proposed method achieved a higher accuracy. In addition, the feasibility of the proposed approach is experimentally verified on the $\mathrm{AE}$ source localization system. The experiment results show that the mean localization error of $3.64 \mathrm{~mm}$ and the standard deviation of $2.61 \mathrm{~mm}$ are obtained, which are $67.55 \%$ and $75.46 \%$ higher than those of the traditional method.

Although the proposed approach realizes the localization of the $\mathrm{AE}$ sources in the structural material, there are still some limitations. For example, if there are untrained normalized amplitude values of the collected AE signals, there will be error location predictions. In future work, we will further expand the $\mathrm{AE}$ signal training dataset and use more novel machine learning methods to optimize the AE source localization approach.

\section{Data Availability}

The data used to support the findings of this study are available from the corresponding author upon request.

\section{Conflicts of Interest}

The authors declare that they have no conflicts of interest.

\section{Acknowledgments}

The authors are grateful for the support from the National Natural Science Foundation of China (61901191, 62073196) and the Natural Science Foundation of Shandong Province (ZR2020LZH005).

\section{References}

[1] S. Jinachandran, Y. Ning, B. Wu et al., "Cold crack monitoring and localization in welding using fiber Bragg grating sensors," IEEE Transactions on Instrumentation and Measurement, vol. 69, no. 11, pp. 9228-9236, 2020.

[2] T. Kundu, “Acoustic source localization," Ultrasonics, vol. 54, pp. 25-38, 2014.

[3] D. Liang, S. Yuan, and M. Liu, "Distributed coordination algorithm for impact location of preciseness and real-time on composite structures," Measurement, vol. 46, pp. 527-536, 2013.

[4] T. Fu, Y. Liu, K. T. Lau, and J. Leng, "Impact source identification in a carbon fiber reinforced polymer plate by using embedded fiber optic acoustic emission sensors," Composites Part B Engineering, vol. 66, pp. 420-429, 2014.

[5] D. Aljets, A. Chong, S. Wilcox, and K. Holford, "Acoustic emission source location on large plate-like structures using a local triangular sensor array," Mechanical Systems and Signal Processing, vol. 30, pp. 91-102, 2012.

[6] J. Tang, W. Lu, Z. Li, and F. Chu, "Acoustic emission localization on a large anisotropic composite plate," in 2015 IEEE International Instrumentation and Measurement Technology Conference (I2MTC) Proceedings, pp. 204-209, Pisa, Italy, 2015.

[7] S. Yin, Z. Cui, and T. Kundu, "Acoustic source localization in anisotropic plates with "Z" shaped sensor clusters," Ultrasonics, vol. 84, pp. 34-37, 2018.

[8] F. Ciampa and M. Meo, "A new algorithm for acoustic emission localization and flexural group velocity determination in anisotropic structures," Composites Part A Applied Science and Manufacturing, vol. 41, no. 12, pp. 1777-1786, 2010.

[9] R. Ye, Q. Wang, X. Fan, and W. G. Zhang, "Acoustic emission source location of composite materials using one channel sensor based on time reversal method," Journal of Wuhan University of Technology, vol. 36, no. 12, pp. 22-26, 2014.

[10] T. He, D. Xiao, Q. Pan, X. Liu, and Y. Shan, "Analysis on accuracy improvement of rotor-stator rubbing localization based on acoustic emission beamforming method," Ultrasonics, vol. 54, pp. 318-329, 2014.

[11] T. He, Q. Pan, Y. Liu, X. Liu, and D. Hu, "Near-field beamforming analysis for acoustic emission source localization," Ultrasonics, vol. 52, no. 5, pp. 587-592, 2012.

[12] F. Ciampa and M. Meo, "Acoustic emission localization in complex dissipative anisotropic structures using a onechannel reciprocal time reversal method," Acoustical Society of America, vol. 130, no. 1, pp. 168-175, 2011.

[13] H. Yang, Y. J. Lee, and S. K. Lee, "Impact source localization in plate utilizing multiple signal classification," Proceedings of the Institution of Mechanical Engineers, Part C: Journal of 
Mechanical Engineering Science, vol. 227, no. 4, pp. 703-713, 2013.

[14] Z. Sharif-Khodaei, M. Ghajari, and M. H. Aliabadi, "Determination of impact location on composite stiffened panels," Smart Materials and Structures, vol. 21, no. 10, article 105026, 2012.

[15] T. Fu, Z. Zhang, Y. Liu, and J. Leng, "Development of an artificial neural network for source localization using a fiber optic acoustic emission sensor array," Structural Health Monitoring, vol. 14, no. 2, pp. 168-177, 2015.

[16] A. D. Deng, X. D. Zhang, J. N. Tang, L. Zhao, and K. Qin, "Localization of acoustic emission source based on chaotic neural networks," Applied Mathematics \& Information Sciences, vol. 6, no. 3, pp. 713-719, 2012.

[17] Q. Liu, L. Wu, F. Wang, and W. Xiao, "A novel support vector machine based on hybrid bat algorithm and its application to identification of low velocity impact areas," IEEE Access, vol. 8, pp. 8286-8299, 2020.

[18] L. De Marchi, A. Marzani, N. Speciale, and E. Viola, "A passive monitoring technique based on dispersion compensation to locate impacts in plate-like structures," Smart Materials and Structures, vol. 20, no. 3, article 035021, 2011.

[19] D. Xiao, T. He, Q. Pan, X. Liu, J. Wang, and Y. Shan, "A novel acoustic emission beamforming method with two uniform linear arrays on plate-like structures," Ultrasonics, vol. 54, no. 2, pp. 737-745, 2014.

[20] L. Dong, X. Li, L. Tang, and F. Gong, "Mathematical functions and parameters for microseismic source location without premeasuring speed," Chinese Journal of Rock Mechanics and Engineering, vol. 30, pp. 2057-2067, 2011.

[21] L. Dong, Q. Hu, X. Tong, and Y. Liu, "Velocity-free MS/AE source location method for three-dimensional holecontaining structures," Engineering, vol. 6, no. 7, pp. 827$834,2020$.

[22] W. Fang, L. Liu, J. Yang, and A. Shui, "A non-iterative AE source localization algorithm with unknown velocity," Journal of Traffic and Logistics Engineering University, vol. 32, pp. 1-7, 2016.

[23] Z. Zhou, R. Lan, Y. Rui et al., "A new acoustic emission source location method using tri-variate kernel density estimator," IEEE Access, vol. 7, pp. 158379-158388, 2019.

[24] Z. Zhou, R. Lan, Y. Rui, L. Dong, and X. Cai, “A new algebraic solution for acoustic emission source localization without premeasuring wave velocity," Sensors, vol. 21, p. 459, 2021.

[25] A. Ebrahimkhanlou and S. Salamone, "Single-sensor acoustic emission source localization in plate-like structures using deep learning," Aerospace, vol. 5, p. 50, 2018.

[26] W. Suwansin and P. Phasukkit, "Deep learning-based acoustic emission scheme for nondestructive localization of cracks in train rails under a load," Sensors, vol. 21, p. 272, 2021.

[27] A. Ebrahimkhanlou, B. Dubuc, and S. Salamone, "A generalizable deep learning framework for localizing and characterizing acoustic emission sources in riveted metallic panels," Mechanical Systems and Signal Processing, vol. 130, pp. 248-272, 2019.

[28] Z. Liu, Q. Peng, X. Li, C. F. He, and B. Wu, "Acoustic emission source localization with generalized regression neural network based on time difference mapping method," Experimental Mechanics, vol. 60, no. 5, pp. 679-694, 2020.
[29] E. D. Niri and S. Salamone, "A probabilistic framework for acoustic emission source localization in plate-like structures," Smart Materials \& Structures, vol. 21, no. 3, article 035009, 2012.

[30] D. Pang, Q. Sui, M. Wang, Y. Sai, R. Sun, and Y. Wang, "Acoustic emission source localization system using fiber Bragg grating sensors and a areal coordinate-based algorithm," Journal of Sensors, vol. 2018, Article ID 9053284, 8 pages, 2018.

[31] Y. Diao, Z. Lin, and M. Fu, "A barycentric coordinate based distributed localization algorithm for sensor networks," IEEE Transactions on Signal Processing, vol. 62, no. 18, pp. 47604771, 2014.

[32] B. Schechinger and T. Vogel, "Acoustic emission for monitoring a reinforced concrete beam subject to four-point-bending," Construction and Building Materials, vol. 21, no. 3, pp. 483490, 2007.

[33] A. H. Seno and M. H. Aliabadi, "Impact localisation in composite plates of different stiffness impactors under simulated environmental and operational conditions," Sensors, vol. 19, no. 17, p. 3659, 2019.

[34] Y. Sai, X. Zhao, L. Wang, and D. Hou, "Impact localization of CFRP structure based on FBG sensor network," Photonic Sensors, vol. 10, no. 1, pp. 88-96, 2020.

[35] B. W. Jang and C. G. Kim, "Impact localization of composite stiffened panel with triangulation method using normalised magnitudes of fiber optic sensor signals," Composite Structures, vol. 211, pp. 522-529, 2019.

[36] M. S. Hossain, Z. C. Ong, Z. Ismail, S. Noroozi, and S. Y. Khoo, "Artificial neural networks for vibration based inverse parametric identifications: a review," Applied Soft Computing, vol. 52, pp. 203-219, 2017.

[37] D. Kolar, D. Lisjak, M. Pajk, and M. Gudlin, "Intelligent fault diagnosis of rotary machinery by convolutional neural network with automatic hyper-parameters tuning using Bayesian optimization," Sensors, vol. 21, no. 7, p. 2411, 2021. 